\title{
FAMILISMO, ASITENCIALISMO Y PRECARIEDAD. LA CONFIGURACIÓN DEL EMPLEO EN EL SECTOR DE ATENCIÓN A LAS PERSONAS EN ESPAÑA
}

\author{
Familism, Welfarism and Precariousness. How \\ Employment is Structured in Spain's Care Sector
}

Carolina Recio Cáceres*

\begin{abstract}
Resumen
El sector de atención a las personas en España ha adquirido una mayor importancia a raíz de la aprobación de la Ley de Dependencia a finales del 2006. La ley, además de reconocer el derecho a recibir cuidados, también considera que éstos deben ser prestados con calidad. Partiendo de este contexto se propone una mirada sobre el empleo en este sector, teniendo en cuenta que las nuevas leyes y normativas hacen preveer un aumento paulatino en la ocupación. Para ello se realiza un análisis amplio que tiene en cuenta las distintas esferas sociales que intervienen en la expansión de un modelo u otro de ocupación en este sector. Se utiliza así la perspectiva sobre los modelos de empleo que analiza el empleo mediante la interacción entre distintas instituciones sociales. Bajo esta perspectiva, el artículo analiza las interacciones entre el modelo familista español, el modelo asistencialista de bienestar y un mercado laboral marcado por la precariedad. Es por ello que el texto plantea que el empleo en el ámbito de la dependencia se explica mediante la relación establecida entre familismo, asistencialismo y precariedad.
\end{abstract}

Palabras clave: cuidados, división sexual del trabajo, empleo, políticas sociales, dependencia.

\footnotetext{
* Carolina Recio Cáceres

Universitat Autònoma de Barcelona

Facultat de Ciències Polítiques i Sociologia

Edifici B. 08193 Cerdanyola del Vallès, Barcelona

Teléfono: 9358133 03/676 807682 Fax: 935812405

carolina.recio@uab.cat
} 


\begin{abstract}
The care sector in Spain has come a long way since the Dependent Care Law came into effect in late 2006. It not only recognises the right to receive public care, but also makes it clear that the care provided should be one of quality. It is in this context that this paper considers employment in the care sector, taking into account that the new laws and regulations mean that a gradual increase in job numbers is to be expected. A broad analysis is made by taking into account the various social spheres involved in expanding various sector employment models. This view of employment models is used to analyse employment by means of the interaction between social institutions - the Spanish family model, the welfare care model and a job market known for its precariousness. This paper therefore considers that employment in the dependent care sector can be explained by the relationship between familism, welfarism and precarious employment.
\end{abstract}

Keywords: care, sexual division of work, employment, social policy, dependent care.

«Lejos de constituir una imagen costumbrista de una tradición feudal y luego burguesa, el empleo de servicio aparece ahora como una necesidad pública y privada a la vista de la evolución económica y social. Paliativo de las carencias del Estado, remedio frente a la desestructuración familiar, expresión de unas nuevas relaciones entre hombres y mujeres, el empleo familiar designa el lugar de una mutación de la organización del trabajo entre la gratuidad y el salario, la intimidad y el salario, la intimidad y la publicidad, la emancipación de las mujeres y la tradición de la función femenina...» (Fraisse, 2000)

\title{
1. Introducción
}

El cuidado se está consolidando como un objeto de estudio recurrente en las Ciencias Sociales, en parte gracias a los trabajos desde la perspectiva de género, y ha ido adquiriendo una mayor atención teórica. Esta atención viene avalada por las realidades de las sociedades occidentales en las que diversos cambios sociales, económicos y culturales, han obligado a los gobiernos a plantear nuevas áreas y formas de acción para dar solución a lo que algunas autoras han bautizado como «la crisis de los cuidados» (Hochschild, 2008; Simonazzi, 2009). El cuidado ya no sólo se presta en el ámbito familiar, ahora también es posible dibujar un escenario en el que el trabajo de cuidados se dibuja como un sector específico de empleo. Este empleo debe ser estudiado desde la relación entre las distintas instituciones sociales, incluyendo elementos propios del contexto social, económico, político y cultural de los distintos países. El objetivo del texto es poner de relieve cómo se configura el empleo en el sector de atención a las personas en España, una propuesta que intenta vincular el ámbito del trabajo y del bienestar en el estudio del cuidado. No 
es sólo estudio de las condiciones de empleo, sino de las relaciones entre esferas sociales que configuran un tipo de modelo de cuidados y que tiene consecuencias sobre el bienestar de la población. Para tal objetivo, el texto se adentra en la perspectiva sobre los modelos de empleo, que pone en relación las distintas instituciones sociales en la concreción de un tipo nacional de empleo o de otro (Rubery, Bosch y Lehndorff, 2008; Bosch, Lehndorff y Rubery, 2009). Esta perspectiva no sólo explica el mercado desde el propio mercado, sino que asume que la acción política o los cambios en la división sexual del trabajo pueden ejercer una presión sobre los modelos productivos nacionales. Además, permite realizar estudios sobre sectores de ocupación concretos, relacionándolos con el modelo de empleo general de una sociedad determinada.

El texto se inicia con un breve repaso de aquellas perspectivas que han permitido visibilizar la importancia de los cuidados para el bienestar de las personas. Y que además nos recuerdan que el cuidado es trabajo. Tras este rápido viaje sobre el concepto de cuidado, nos detendremos en la propuesta de los modelos de empleo y su plasmación en el sector de atención a las personas. Para terminar echaremos una mirada sobre el caso español, y cómo éste parece definirse como un modelo familista, asistencialista y precario, aun asumiendo que se observan algunos indicios que harían pensar en un posible proceso de transición a un modelo distinto.

\section{La construcción sociológica del cuidado}

En las siguientes líneas realizaremos un breve repaso de aquellos trabajos que han contribuido al asentamiento del concepto de cuidado, y que nos va a permitir entender los apartados posteriores. Partiremos de la tesis de Teresa Torns (2008), que defiende el estudio del cuidado desde el ámbito de la sociología del trabajo, y realiza un análisis que conjugue el trabajo con el bienestar. Esta perspectiva reivindica la recuperación de aquellos estudios del cuidado desde el concepto de trabajo y la importancia de su realización para el bienestar cotidiano que permitieron, años después, avanzar en el estudio del cuidado.

\subsection{De la ruptura conceptual al social care}

Los trabajos pioneros en el ámbito del care se inscriben en la literatura científica feminista de la década de los sesenta. Desde el marxismo algunas científicas sociales iniciaron los debates acerca del papel del trabajo de las mujeres en la sociedad, y su relación con el sistema capitalista. En estos trabajos el cuidado no era el núcleo central, sino que la idea del care se incluía dentro de los debates sobre el trabajo no remunerado realizado en el seno de los hogares 
por las mujeres adultas (Letablier, 2007; Torns, 2008). Estos debates nacieron del interés de introducir la relación de explotación hombre-mujer en la teoría marxista (Astelarra, 1982). Siguieron aquellos trabajos que contribuyeron a dotar de estatuto teórico al trabajo doméstico y familiar, que engloba a su vez el trabajo de cuidado de los miembros dependientes de la familia. La revisión del significado del concepto de trabajo supuso el reconocimiento de la actividad invisible que las mujeres realizaban en el seno de los hogares y que era necesaria para la reproducción de la fuerza de trabajo (Benería, 1981; Borderías, Carrasco y Alemany, 1994). Podría situarse dentro de las teorías de la dualidad, en que se reconocen dos esferas, la pública y la privada. La primera puede definirse, grosso modo, por ser masculina, remunerada, valorada y fuente de derechos de ciudadanía. La segunda es femenina, no remunerada, invisible y está desprovista de derechos de ciudadanía (Benería, 1981; Picchio, 1994; Balbo, 1994, Borderías, Carrasco y Alemany, 1994; Rubery, 1997; Gardiner, 2000). Es el modelo de male breadwinner y female housekeeper que supone que el marido es el que mediante las rentas generadas por su trabajo remunerado sustenta económicamente al resto de miembros de la familia, y al mismo tiempo obtiene los beneficios sociales que le otorga su estatus de empleado (Rubery, 1997; Gardiner, 2000). Es importante hacer visibles estas investigaciones puesto que fueron pioneros en el estudio del trabajo, incluyendo la tarea de cuidar dentro del trabajo doméstico y familiar.

El cuidado al que se hacía referencia era el realizado dentro de los hogares, por ello, mucha de la literatura sobre el care se dedicó al estudio del cuidado informal, entendiendo por informal aquel que se realizaba mediante el trabajo no remunerado de las mujeres adultas de las familias (Finch y Groves, 1983; Graham, 1983; Ungerson, 1990; Letablier, 2007). En esta línea encontramos aquellos estudios que introducían la importancia de la emotividad y los sentimientos en el trabajo de cuidados. De hecho, el título de Finch y Groves (1983) es el que mejor resume las posturas en esta línea: A labour of love: work, care and feeling. Desde esta perspectiva consideran que hay trabajo doméstico que puede ser racionalizado y rutinizado, mientras que entienden que el cuidado es una actividad sensiblemente distinta. El cuidado requiere no sólo actividad física, sino también emociones y sentimientos. Definen el cuidado como una actividad material y una relación emotiva (Finch y Groves, 1983; Graham, 1983). Esta consideración del trabajo de cuidados, alejada en parte de la concepción del cuidado como trabajo, es la que ha marcado muchos de los debates pasados y actuales sobre la ética del cuidado, tanto en el ámbito privado del hogar como en los empleos que supuestamente contienen una alta carga emocional. 
En la década de los ochenta, y especialmente en la de los noventa, el interés por el estudio se desplaza al área de la política social. Desde Reino Unido y los países nórdicos llegan los primeros trabajos en esta línea: el estudio del trabajo de cuidados y las políticas públicas que se diseñan en esta línea. En estos países existía algún tipo de políticas públicas en relación a los cuidados de la población. De ahí que las investigadoras de ambas realidades nacionales se interesaran por la temática. Mientras que en los países nórdicos se intentaba delimitar en qué medida los servicios públicos suavizaban la carga de trabajo de las familias, en Reino Unido el traslado del cuidado a la comunidad era el foco principal de atención (Ungerson, 1990). En Reino Unido se estaba produciendo una transición hacia la promoción del care by the comunity, que en la práctica significaba una pérdida del poder público para prestar cuidado, trasladándose los costes a la comunidad. En este contexto se desarrollaron con fuerza los análisis desde la política social. El envejecimiento de la población, la mayor presencia de las mujeres en el mercado de trabajo, los cambios culturales que supuso el avance de los movimientos feministas, explican también por qué el cuidado pasa a ser un foco de interés en las Ciencias Sociales. Así, las exigencias del cuidado de las personas dependientes se hicieron visibles. También, ante la proliferación de estudios comparativos de los distintos Estados del Bienestar, las autoras feministas adoptaron una perspectiva nueva, que ponía el cuidado en el centro de los análisis. Se entiende que el cuidado es una parte inseparable del bienestar de las personas, y por ello es necesario introducirlo en los análisis de los Estados del Bienestar. Reconocen la tarea invisible que han realizado las mujeres en el seno de los hogares y su importancia capital en el estudio de los Estados del Bienestar (Carrasco et al, 1997, Sainsbury, 1996; Daly y Lewis, 2000; Letablier, 2007).

Con este trasfondo, el care obtiene estatuto teórico y aparecen propuestas consistentes para obtener una definición del concepto, siempre teniendo en cuenta su naturaleza relacional. Se intenta captar desde el cuidado informal al formal, el papel de los poderes públicos, la persistencia de la división sexual del trabajo en los hogares, etc. Carole Thomas (1993) y posteriormente Daly y Lewis (2000) son algunas de las aportaciones más destacadas y que han obtenido más reconocimiento en las Ciencias Sociales. A ambas se las debe entender como un avance definitivo en el campo de estudio del care, específicamente del social care, o lo que en castellano podríamos traducir como las formas de organizar socialmente los trabajos de cuidados. Hacen referencia pues a las relaciones entre actores e instituciones y los modelos de cuidado que de éstas se derivan (Letablier, 2007). Construyen el concepto de social care que les ha de permitir la realización de estudios comparativos entre distintas realidades 
nacionales. Las dimensiones propuestas se materializan en tres aspectos. En primer lugar reconocen que el care es trabajo, considerando todas las formas en que se realiza, lo cual significa que rompen la tendencia dicotómica en el estudio del cuidado. En segundo lugar deben considerarse los marcos normativos en los que se realiza el cuidado, es decir, se debe atender a las responsabilidades y obligaciones que marcan las distintas sociedades en relación al cuidado. Por ejemplo, hay estados que pueden reconocer la obligación a las familias a realizar trabajos de cuidados a los miembros de su familia, mientras que otros no recogen esta obligación. En tercer lugar, aseguran que el concepto del social care permite medir los costes materiales, financieros y emocionales que causa la actividad de cuidar. Reconocían además que el estudio de los regímenes de cuidado debía incluir tanto el cuidado de menores como el cuidado de adultos, pensando en el aumento imparable de la población anciana. Este cambio demográfico debía contar con estructuras conceptuales capaces de captar los cuidados que requieren los distintos colectivos de edad, que suelen esconder grandes diferencias en términos de necesidades de cuidado. Además, esta propuesta permitía no sólo avanzar en el conocimiento sobre las condiciones de vida de las mujeres, sino que también incluía los elementos necesarios para captar las necesidades sociales y personales que conducen al bienestar.

Esta construcción teórica les valió para desarrollar una nueva propuesta sobre los modelos de Estados del Bienestar, que se oponía claramente a todas las que habían omitido el género en sus análisis. Otras autoras han seguido esta línea inaugurada por las autoras anglosajonas, Bettio, Simonazzi y Villa (2006), y consideran necesario atender tanto a las políticas sociales como a la historia cultural de los países en cuestión. Todas son propuestas que ahondan en el concepto de ciudadanía que ya no sólo se fijará en la presencia pública de las personas, sino que añadirá también otras esferas de la vida. (No sólo el empleo es fuente de bienestar). Estas consideraciones se traducen en regímenes de cuidado que tratan de dilucidar cuál es el sistema de organización social de los cuidados en los distintos países. Cabe destacar que en este sentido se recoge el tipo de servicios generadas, y es precisamente lo que aquí se pretende estudiar. El tipo de empleo creado depende del lugar que ocupa el cuidado en las políticas encaminadas al bienestar de la población, especialmente porque el cuidado se convierte en derecho de ciudadanía. Esto implica reconocer la necesidad de un cuidado profesional, ya que las personas dependientes necesitan unas atenciones concretas. Si esto es lo que se desprende de estas reflexiones, veamos qué es lo que está sucediendo en la práctica, y dónde se hallan las posibilidades para lograr este objetivo. 


\section{Los modelos nacionales de empleo}

El concepto de modelo de empleo sugiere el análisis de la actividad laboral desde una perspectiva relacional, o si se prefiere desde una lógica institucional. Esto significa que los empleos bajo esta perspectiva analizan los patrones de desarrollo de los mercados de trabajo teniendo en cuenta no sólo el análisis de las variables del mercado laboral, sino las interrelaciones de éste con las políticas sociales, educativas, laborales y económicas. El modelo analítico propuesto incluye por tanto variables mercantiles y no mercantiles y relaciona la «institución» laboral con otras instituciones sociales extra-mercado que configuran la vida laboral de las personas (Bosch, Lehndorff y Rubery, 2009). En general, el estudio de los modelos de empleo plantea un análisis que interrelaciona tres espacios de la vida social: el sistema productivo, el sistema público y el sistema familiar. En otras palabras, parte del modelo «Mercado, Estado, Familia». Además, la perspectiva requiere un análisis de carácter histórico capaz de captar los cambios acaecidos en los modelos de empleo. Es decir, que los modelos nacionales de empleo son el resultado de procesos históricos que van configurando y/o transformando los sistemas en cada momento.

"It is the employment relationship embedded within this multiplicity of interlocking institutions that give rise to what we call nacional employment models. (...) This interlocking of institutional arrangments also creates the possibility of spillover effects from one sphere to another, potentially increasing the scope of change through ripple of domino effects. Thus change to the nature of the internalized employment relationship has implications for the survival of the welfare system for change within the employment model may originate in different spheres» (Bosch, Lehndorff y Rubery, 2009: 2)

El espacio mercantil hace referencia a los sistemas productivos nacionales, donde es importante tener en cuenta el papel central de las empresas capitalistas y las regulaciones específicas del mercado de trabajo. Se debe tener en cuenta tanto las influencias, a nivel local, de los procesos de integración económica mundial, como las acciones de los propios empresarios en relación con las estrategias de especialización y organización. También debe considerarse el sistema de relaciones laborales. El espacio familiar atiende a la división sexual del trabajo dentro del seno de los hogares, y al peso de las familias en la proporción de bienestar, puesto que hay realidades donde el trabajo doméstico y familiar suple en muchos casos la acción del sistema público y limita o modula la presencia de las personas en el espacio mercantil. El espacio público está definido por el conjunto de políticas públicas y, por tanto, incluye el sistema de provisión pública de bienes y servicios. Unas políticas que también 
influirán en los otros dos espacios (Bosch, Lehndorff y, Rubery, 2009; Banyuls et al, 2009).

Otro elemento importante de esta perspectiva es que permite realizar estudios comparativos entre los distintos modelos nacionales. Este planteamiento supera, en parte, otras propuestas sobre modelos comparados entre países, ya sea desde la lógica productiva -los trabajos sobre variedades de capitalismo (Coates, 2005) - como desde la lógica de los Estados del Bienestar (Esping-Andersen, 1990). En lugar de fijarse tan sólo en la acción de una de las instituciones sociales, pone en diálogo a tres espacios sociales. Y, además, tiene en cuenta tanto los factores externos (políticas europeas, procesos migratorios, entre otros) como los factores internos (envejecimiento de la población, incorporación de las mujeres al mercado laboral, etc.). En parte, los trabajos de modelos nacionales de empleo nacieron como una forma de ver hasta qué punto se podía afirmar que era posible hablar del modelo social europeo (Rubery, Bosch y Lehndorff, 2008), y de cómo los países tratan de amoldarse o no a él. En este sentido, los primeros resultados de algunas investigaciones dedicadas al estudio de estos procesos ${ }^{1}$ apuntan a que si bien hay directrices comunes, basadas en el modelo social europeo, cada país ha elaborado sus propias vías de desarrollo, en función de tradiciones económicas, políticas y sociales propias.

Finalmente, la perspectiva de los modelos nacionales de empleo permite el estudio de sectores de empleo concretos. De hecho, tomando como punto de referencia el modelo general, es posible realizar el estudio del sector concreto del cuidado. En este caso, el modelo de empleo generado en el sector remite a un entresijo de relaciones establecidas entre los distintos espacios institucionales descritos. Las distintas formas de concebir el propio trabajo, las políticas sociales y el empleo dibujan un tipo u otro de sector, es decir, se configurará un sector más o menos formal (en el sentido de mayor o menor utilización de la economía sumergida), con mayor o menor reconocimiento de las profesiones vinculadas al mismo, con mayores o menores problemas para reclutar mano de obra, etc. En definitiva, el análisis derivado de ello defiende que el sistema de cuidados de un país concreto se debe explicar por la relación entre modelo laboral, modelo familiar y modelo de Estado. Unas consideraciones que mostraremos en el siguiente apartado.

1. Véanse los resultados del proyecto «Dynamics of National Employment Models» (Dynamo), financiado por la Comision Europea (VI Programa Marco) y coordinado por el profesor Steffen Lehndorff. Algunas de las reflexiones de los hallazgos del proyecto pueden encontrarse en Bosch, Lehndorff y Rubery, 2009. 


\section{El sector de atención a las personas: regímenes de empleo y regímenes de cuidado}

El sector de atención a las personas se está desarrollando rápidamente en la gran mayoría de países europeos. Los cambios sociodemográficos han obligado a los Estados a diseñar distintas acciones en relación a la necesidad de cuidados por parte de la población dependiente. La expansión de este sector no sólo se explica por la ampliación de los servicios públicos, sino que también intervienen los tímidos cambios en la división sexual del trabajo, la incorporación de la mujer al mercado de trabajo, la necesidad de crear empleo en nuevos sectores productivos, etc. Por tanto, para poder estudiar este sector es necesario atender a los cambios sociales, económicos y culturales acontecidos en los distintos países europeos, especialmente a partir de los años noventa (Rubery, Smith y Fagan, 1999). Estos cambios están detrás de la configuración de un tipo u otro de mercado del care. Las relaciones establecidas entre uno y otro aspecto interactúan con las posibilidades y las formas en que se va a desarrollar el sector del cuidado, y el tipo de empleo que se va a generar (Simonazzi, 2009).

Los trabajos de Anna Simonazzi (2007 y 2009) suponen un avance en el campo de estudio de los sectores de ocupación relacionados con el care, partiendo de la perspectiva analítica de los modelos nacionales de empleo. Así, las tesis de Simonazzi apuntan que un tipo u otro de regímenes de care (políticas sociales destinadas a atender a la población dependiente) generan un tipo específico de mercado en este sector: "The way in which elderly care is provided and financed may entail considerable differences in the creation of formal care market» (Simonazzi, 2009: 211). El sector de empleo vinculado a la prestación de cuidados debe relacionarse tanto con las políticas públicas como con las tendencias propias de los mercados de trabajo nacionales y con la tradición familiar de los distintos países, que se encuentra definida mediante el reconocimiento formal y legal de las obligaciones de las familias en los cuidados de las personas dependientes (Anxo, Fagan, 2005; Fagan, Nixon, 2001; Simonazzi, 2009). La interacción entre estos elementos generará un tipo u otro de empleo en el sentido no sólo de la cantidad del empleo generado, sino también de su calidad. Todo ello genera unas prácticas y un modelo distinto de mercado de trabajo en el sector de atención a la dependencia. Aquellos sistemas que apuestan por la prestación de servicios públicos y/o las transferencias monetarias «bajo control» son aquellos que más posibilidades generan de crear un mercado formal en el ámbito del social care. Mientras que aquellos Estados que apuestan por transferencias económicas sin comprobación y/o trasladan a las familias un volumen más elevado de obligaciones 
también están depositando su confianza en un sistema informal de cuidados (ya sea mediante la prestación familiar o bajo contrataciones propias de la economía sumergida). Desde este conjunto de relaciones debe estudiarse el empleo en este sector. Los salarios, las condiciones de trabajo, la formación y habilidades requeridas son elementos que pueden dirigir hacia empleos de calidad o, por el contrario, delatar la precariedad de los sectores (Aragón, Cruces y Rocha, 2008). Pero en la determinación de estas condiciones debe tenerse en cuenta la acción empresarial, los procesos de relaciones laborales (espacio mercantil), el tipo de promoción pública de los servicios y el modelo de despliegue de los servicios (espacio público) y la persistencia o superación del modelo male breadwiner y female housekeeper (espacio familiar).

Los distintos países de la Unión Europea han diseñado estrategias distintas, que se relacionan con lo expuesto en el párrafo anterior (Anxo, Nyman y Fagan, 2001; Moss, 2004; Simonazzi, 2007 y 2009)². Por ejemplo, en el caso sueco, la apuesta ha sido generar ocupaciones cualificadas y con unas condiciones relativamente buenas, en parte por su inclinación por un modelo de políticas universales y de servicios públicos de atención a las personas, y una fuerte influencia de las políticas de igualdad de género (Evertsson, 2000; Anxo y Nyman, 2001; Ahmed, 2006). Otro ejemplo paradigmático podría ser el de Reino Unido. Se trata de un Estado que hace años que empezó a desarrollar los servicios de atención a las personas. También, como en el caso sueco, se han fomentado los servicios de atención a las personas, pero al contrario que el país escandinavo, la estrategia ha sido la de expansión de un sector precario, de bajos salarios y sin necesidades de formación específicas en el momento de entrada en el sector (Fagan y Nixon, 2001). El modelo anglosajón se asienta sobre un modelo de familia «ausente», en el sentido de que no existe ninguna obligación normativa que exija cuidar a los demás miembros de la familia; en un Estado que sí ha previsto distintas políticas de atención a las personas dependientes, pero que dejan mucho espacio al desarrollo de las empresas privadas y a las entidades del Tercer Sector.

Finalmente, y por citar un caso más cercano, están los países del sur de Europa, donde existe una fuerte tradición familista, unos servicios sociales escasos y una mayor tendencia a las transferencias monetarias. En ellos se observa un mercado laboral de cuidados precario, con condiciones de empleo similares a los que apuntábamos para el caso anglosajón. El resultado es un empleo caracterizado por los bajos salarios, las jornadas laborales largas y/o

2. Los resultados del proyecto «Dynamics of National Employment Models» (Dynamo) financiado por la Comision Europea (VI Programa Marco) y coordinado por el profesor Steffen Lehndorff. Son especialmente relevantes los resultados en el área del care sector. 
atípicas y las pocas posibilidades de promoción profesional, que funcionan como elementos de desmotivación. Y, en el caso de los países del sur de Europa, esta escasez se está solucionando mediante el traslado de obligaciones a las mujeres adultas de las familias o hacia la contratación de mano de obra inmigrante femenina (Bettio, Simonazzi yVilla, 2006; Simonazzi, 2009).

\section{El caso español: familismo, asistencialismo y precariedad}

La perspectiva de los modelos de empleo defiende que España se encuentra en la encrucijada, puesto que se reconocen rasgos heredados de la dictadura franquista, posteriormente moldeados durante el proceso de transición cuando se creó un sistema de servicios y beneficios sociales públicos y universales, pero que pronto recibieron la influencia de la política económica de corte neoliberal, marcando definitivamente el modelo español de empleo. También cabe resaltar una tradición familista, un peso importante de la Iglesia católica y una política social a medio camino entre el universalismo y el asistencialismo (Banyuls et al., 2009). Tras las primeras elecciones democráticas es posible distinguir distintos cambios que afectaron a la estructura económica y social, la cultura, las relaciones laborales y los roles de género. Las causas de estos procesos de cambio deben buscarse tanto en procesos externos (globalización, influencia de la política común europea) como en procesos internos (democratización del país, reestructuración territorial, demandas sociales y cambios culturales) (Banyuls et. al, 2009). En este esquema, el modelo de partida era el de una economía muy cerrada, con una intervención pública (propia de un sistema de dictadura como el franquista), con un bajo nivel de desarrollo de las prestaciones sociales y una familia tradicional, en la que la norma era la provisión del bienestar mediante el trabajo de las mujeres adultas de las familias. Los cambios en los años ochenta de dos fuerzas distintas y a la vez contradictorias son los que pueden dar cuenta del modelo español. En primer lugar, la influencia de las políticas económicas neoliberales que se pusieron de parte del capital y limitaron el gasto público; del eje contrario venían las demandas sociales para la mejora del bienestar de la población, y que ejercieron una fuerza importante para el desarrollo de servicios de bienestar, regulaciones laborales, pensiones, etc. Entre las demandas destaca la del movimiento feminista, que en parte defendió la entrada al mercado laboral como la principal vía de la emancipación de la mujer (Banyuls et al., 2009). Si bien es muy destacable el incremento de la presencia de mujeres en el mercado laboral, no parece tan claro que se haya roto con la fuerte división sexual del trabajo, y los trabajos domésticos y familiares siguen recayendo hoy en día y de forma mayoritaria en las mujeres adultas (Torns et al., 2006; Borras, 
Moreno y Recio, 2009). Unas demandas que también se han hecho oír en el ámbito de la política social. Este proceso, que ha marcado de forma definitiva el momento actual, se ha dado en una sociedad que ha cambiado sustancialmente. Hablamos de una sociedad que envejece y que ha asistido a un cambio en la presencia de las mujeres en el mercado laboral y una persistencia de la ausencia masculina en el trabajo doméstico y familiar, o el paso de ser un país emisor de población a ser un país receptor de población extranjera (Torns et al., 2009; Borras, Moreno y Recio, 2009). También, en la actualidad, nos encontramos con una expansión de los servicios que dan cuenta de estos cambios, especialmente en el campo de las necesidades de cuidado a las personas, una evidencia que recoge la aprobación de la Ley de Promoción de la Autonomía Personal y Atención a las personas en situación de Dependencia, de 14 de diciembre de 2006, o de otras normativas autonómicas como puede ser el caso catalán y la Llei de Serveis Socials. En este contexto social se configura el sector de atención a las personas, y de aquí en adelante plantearemos los tres ejes que modulan el sector.

\subsection{Familia, Estado y mercado}

\subsubsection{La persistencia de la división sexual del trabajo}

En referencia a la familia se distingue una persistencia del modelo male breadwinner, pero no tanto de la mujer dedicada al trabajo doméstico y familiar exclusivamente, sino más bien de mujeres que viven en un Estado de «doble presencia» (Balbo, 1994). Entre los cambios más destacados se deben citar las mayores tasas de actividad de las mujeres y el cambio sensible de las trayectorias laborales de las mujeres, especialmente el de las generaciones jóvenes. Éstas parecen dibujar una trayectoria laboral distinta a la de sus madres y abuelas: abandonan menos el mercado laboral en el momento del matrimonio y la maternidad (Torns et al., 2009). Aun así la división sexual del trabajo es acusada, y son ellas, con mucha diferencia, las protagonistas del trabajo doméstico y familiar (Borrás et al., 2009). Esta perpetuación de la división sexual del trabajo no sólo se expresa en las prácticas cotidianas, sino también mediante los valores e imaginarios sociales, que todavía hoy siguen basándose en un modelo de familia tradicional en el que el hombre es el cabeza de familia, y la mujer, la responsable de los cuidados. Una encuesta realizada por el IMSERSO ${ }^{3}$ en el 2004 preguntaba a personas cuidadoras acerca de sus

3. Ver IMSERSO, Cuidados a las personas en los hogares españoles. El entorno familiar, MTASIMSERSO, Madrid, 2005, [en línea], <http://www.seg-social.es/imserso/documentación/ cuidadosppmmhogares.pdf>. 
preferencias en un hipotético futuro sobre su necesidad de recibir cuidados. Los resultados sobre las preferencias de cómo recibir los cuidados indican que las personas entrevistadas preferirían mayoritariamente recibir los cuidados por parte de algún miembro de la propia familia. Un 25,7 \% expresaba el deseo de ser cuidado/a por una hija, un 19,3\% esperaba ser cuidado/a por el esposo o esposa y un 13,8 \% por un hijo o hija indistintamente. La opción de recibir los cuidados a través de un hijo sólo se consideraba en un 5,4\% de los casos. Las opciones sobre los servicios y/o cuidados externos a los recursos familiares obtenían unos porcentajes mucho más tímidos. Y, cabe destacar que las primeras opciones contempladas demuestran la preferencia de la atención prestada por el sector público en detrimento del sector privado. También desde el punto de vista de los imaginarios hay que resaltar las creencias sociales en torno al significado del trabajo de cuidados, un trabajo asociado a un trabajo servil, desprovisto de valor social, y que supone vivir en una situación de desigualdad para aquellas personas que lo realizan (Fraisse, 2000). Asimismo, la Iglesia católica ha jugado un papel muy importante en el campo ideológico, formando parte de la política nacional durante la época franquista, y que tras la transición la sociedad no supo romper definitivamente con las doctrinas que esta institución insuflaba. Su defensa acérrima de la familia tradicional avala esta tesis. Todo conlleva a una situación en que las mujeres son las principales proveedoras del trabajo de cuidados y que éste es visto, comúnmente, como «el buen cuidado». Más adelante defenderemos la idea que las nuevas políticas públicas han diseñado sobre la base de una sociedad familista (la familia sigue siendo el principal mecanismo de soporte social y de proporción de bienestar). Un modelo que tiene consecuencias claras en el ámbito del empleo y que puede entorpecer los intentos de generar un sistema profesional de atención a las personas.

5.1.2. Las políticas sociales de bienestar: entre el discurso universalista y las prácticas asistencialistas

En el área de las políticas sociales es posible distinguir una doble vía: universalista y asistencialista. Desde el área que nos interesa, diremos que se ha avanzado en materia de derechos sociales, pero como podemos ver éstos aún entrañan una fuerte dependencia con el modelo familiar español y limitan el desarrollo de un mercado de cuidado pensado desde la calidad y el bienestar. En este sentido, persisten los grandes sistemas universales como pueden ser la Sanidad, la Educación y el sistema de pensiones, pero simultáneamente han ido avanzando con una cierta lógica privatizadora, ya que en muchos casos subsisten con estructuras paralelas privadas y/o han visto introducidas 
medidas de gestión privada (Pastor, 2001 y 2006). Estas medidas se concretan en la introducción de la filosofía del management en la organización del trabajo desde la propia institución, o la externalización de alguna de las fases del servicio, especialmente en la fase de gestión del mismo (Rodríguez Cabrero, 1991 y 2002). Las justificaciones a este doble proceso de privatización-externalización nacen de las críticas neoliberales a la excesiva intervención del Estado keynesiano. Unas justificaciones que defendían que la privatización era sinónimo de eficiencia y eficacia y reducía los costes y los trámites burocráticos. Un discurso que caló rápidamente y facilitó la puesta en escena de este tipo de políticas sin generar excesivas resistencias entre el conjunto de la población (Muñoz de Bustillo, 2000; Pastor, 2006). Esto encaja con el escaso aumento del gasto público en España, que apenas ha ascendido en los últimos diez años y se ha situado por debajo de la media de la Unión Europea: el gasto social en España en 2005, y en porcentaje del PIB, fue del 20,8 \% frente al $27,2 \%$ del conjunto de la UE- 27 , o el 27,8 \% de la UE-15. También, se debe destacar la impopularidad de realizar políticas impositivas más decididas que son las que en última instancia permitirían un mayor avance del gasto dedicado a políticas de bienestar.

El sector de atención a las personas se ha desarrollado con fuerza en los últimos años, pero coincide con algunos de los aspectos generales descritos para el conjunto de políticas y servicios sociales de la sociedad española. Antes se trataba de un servicio asistencial y que apenas era conocido por la población en general. Hoy en día se ha invertido algo está realidad ya que los servicios de atención a la dependencia se piensan desde la filosofía universalista, aunque ésta permanece más a nivel político-discursivo que no a nivel de prácticas reales. Veámoslo. En primer lugar, estos servicios están sujetos a la lógica de la privatización-externalización: la titularidad es pública y la gestión del servicio está en manos de empresas privadas. Una tendencia que están aprovechando las grandes empresas para desarrollar nuevas áreas de inversión, en un negocio que entraña poco riesgo, pues estos servicios parecen estar expandiéndose. Muchas de ellas provienen de otros sectores, especialmente del de la construcción, que han podido invertir en estas actividades y que se auguran un negocio seguro en este sector (Miguélez, Lope y Olivares, 2006). Aunque no está de más apuntar que juntamente con estos grandes grupos coexiste un numeroso grupo de empresas locales y relativamente pequeñas que ofrecen este tipo de servicios. Muchas de ellas ligadas al tercer sector, que desde hace muchísimos años trabajan con gente desvalida y marginal, pero también se

4. Ver EUROSTAT. 
trata de pequeñas empresas ligadas a los ámbitos locales (Recio, 2009)5. Este sistema supone que son las Administraciones Locales las que controlan el servicio y fijan su precio público. Las políticas públicas pues juegan un papel esencial para entender la fijación de condiciones de empleo en este sector, como por ejemplo en las políticas salariales.

Si atendemos al tipo de servicios hay que reconocer los avances realizados en las últimas décadas, especialmente en los últimos tres años cuando se han aprobado las distintas normativas que han impulsado definitivamente el sistema de atención a las personas, y han facilitado la expansión y diseño de un sistema público que atiende a la necesidad de cuidados. Hoy todavía es pronto para realizar un análisis sobre el impacto de estas normativas, pero sí es posible extraer algunas reflexiones al respecto que van a tener gran influencia en la configuración del empleo en el sector. En primer lugar, y en sintonía con el resto de países europeos, el discurso político promueve la expansión de los servicios de atención domiciliaria, ya que se piensa que las personas tienen mayor calidad de vida cuanto menos separadas estén de su contexto cotidiano. Sin embargo, el hecho de que se fomente la permanencia de las personas dependientes en su entorno familiar es una forma de asegurar la persistencia de una institución familiar fuerte pues no se altera su estructura y el reparto desigual de trabajos domésticos y familiares (Bettio, Simonazzi y Villa, 2006), permitiendo además una reducción de los costes económicos que genera el mantenimiento de un recurso residencial. La Tabla 1 muestra

Tabla 1. Tasas de cobertura de los Servicios Sociales.

Población mayor de 65 años. España 2002 y 2008

\begin{tabular}{|l|c|c|c|}
\hline & 2002 & 2008 & Variación 08/02 \\
\hline Servicio de Atención Domiciliaria & 2,75 & 4,69 & $+1,94$ \\
\hline Servicio de Teleasistencia & 1,45 & 4,72 & $+3,27$ \\
\hline Centros de Día (total) & 0,26 & 0,83 & $+0,57$ \\
\hline Plazas públicas + concertadas & 0,14 & 0,53 & $+0,38$ \\
\hline Plazas privadas & 0,12 & 0,30 & $+0,19$ \\
\hline Residencias (total) & 3,34 & 4,31 & $+0,97$ \\
\hline Plazas públicas + concertadas & 1,38 & 2,04 & $+0,65$ \\
\hline Plazas privadas & 1,96 & 2,28 & $+0,32$ \\
\hline
\end{tabular}

Fuente: IMSERSO, Las personas mayores en España. Informe 2008.

5. Ver el estudio de Lope, Recio y Gibert de 1997 en el que se estudió el caso del SAD de Manlleu, y que resulta un buen ejemplo de la existencia de pequeñas empresas privadas y del Tercer Sector muy ligadas a municipios pequeños y medianos. 
que los mayores incrementos en las tasas de cobertura de los servicios públicos de atención a las personas se encuentran precisamente en los SAD y la teleasistencia. Asimismo queda claro que es en el servicio más costoso, el centro residencial, donde la presencia del sector privado es mayor. Según el Informe del IMSERSO sobre las personas mayores, el $87 \%$ de las residencias para la tercera edad son de titularidad privada, frente a un tímido $13 \%$ de titularidad pública.

Después de tres años de andadura de la Ley de Dependencia existen registros que permiten hacer alguna valoración sobre el alcance de dicha ley. Y, empezar a visualizar si éste ha contribuido a construir un Sistema Público de Atención a las Personas. Los datos ofrecidos ponen de relieve que aquellas prestaciones que más se están aprobando son, precisamente, las prestaciones económicas a los cuidados personales (ver Tabla 2), medida prevista con carácter de excepcionalidad y que se está convirtiendo en una normalidad. De este modo, más del $30 \%$ de los Planes Individuales de Atención resuelven que la prestación social a recibir es una transferencia monetaria a la cuidadora principal de la persona dependiente. Esta cuidadora recibirá un salario y será dada de alta en el régimen de la Seguridad Social, contribuyendo así a la disminución de la elevada tasa de desempleo femenino. Las personas que reciben este «salario» son consideradas «cuidadores no profesionales». Un ejemplo que hace pensar que el modelo público de cuidados sigue apoyándose en las mujeres, un elemento que limita la expansión de un sistema realmente profesionalizador.

Tabla 2. Prestaciones reconocidas en el PIA. España.

Datos del 1 de septiembre de 2009

\begin{tabular}{|l|c|c|}
\hline & N & $\%$ \\
\hline $\begin{array}{l}\text { Prevención a la dependencia y promoción A, } \\
\text { Personal }\end{array}$ & 2554 & 0,39 \\
\hline Teleasistencia & 28286 & 4,32 \\
\hline Servicio de Ayuda a Domicilio & 46509 & 7,11 \\
\hline Centros de Día/Noche & 22827 & 3,49 \\
\hline Atención Residencial & 86220 & 13,18 \\
\hline Prestación Económica Vinculada al Servicio & 28633 & 4,38 \\
\hline Prestación Económica a Cuidados Personales & 228817 & 34,99 \\
\hline Prestación para la Asistencia Personal & 630 & 0,10 \\
\hline Total & 444476 & 67,96 \\
\hline Pendientes del PIA & 209559 & 32,04 \\
\hline Total & 654035 & 100 \\
\hline
\end{tabular}

Fuente: IMSERSO/Sistema para la Autonomía y Atención a la Dependencia. 


\subsubsection{El empleo en el sector de atención a las personas}

El mercado laboral español bebería de los dos procesos descritos, de ahí que nos encontremos en un menor desarrollo del sector servicios y un mercado de trabajo muy segmentado por razones de género. En líneas generales, el mercado laboral español se caracteriza por el hiperdesarrollo de ciertos sectores, en detrimento de otros, como son los servicios públicos, que tienen un efecto clave sobre el nivel de desigualdad de una población y por ende sobre su nivel de bienestar. En general, en España se dibuja un mercado definido por estar muy segmentado, y de terminarse precisamente desde la precariedad laboral, con una presencia limitada de las organizaciones sindicales, en parte debido al gran número de pequeñas y medianas empresas que supone un freno a la sindicalización. Los expertos en mercado laboral nos dibujan el caso español como un mercado laboral precarizado, con altas tasas de temporalidad y paro, e inestable (Miguélez y Prieto, 1999; Miguélez, 2004; Banyuls et al, 2009). caracterizado, también por estar muy segmentado por razones de género. Así, las mujeres están sobrerepresentadas en los sectores menos cualificados y con salarios menores (Recio, 2001), es decir, el mercado laboral femenino se caracteriza por su segregación horizontal y su segregación vertical (Torns, 1999). Existen otros elementos a tener en cuenta para entender el mercado de trabajo español y que tienen una clara influencia sobre el sector de atención a las personas. En primer lugar, la recepción de flujos migratorios, de hecho las personas extranjeras han venido por el efecto llamada que se ha producido a raíz de las necesidades de mano de obra del mercado de trabajo español. Los hombres han venido a ocupar, principalmente, los puestos en el sector de la construcción, mientras que muchas mujeres han cubierto las necesidades en el sector de los cuidados. Otra característica que define el mercado de trabajo español y que tiene una importancia esencial en el sector que estudiamos es el modelo formativo. Un mercado que apuesta por el desarrollo de actividades no cualificadas, es decir, ha basado su expansión en actividades que requerían de una escasa formación profesional de entrada; aunque este no requerimiento de formación previa no es un aspecto neutro, sino que tiene que ver con la forma cómo se ha valorado la cualificación necesaria para desempeñar un empleo determinado. El caso del trabajo de atención a las personas es paradigmático en este sentido, ya que no es que no se trate de trabajo cualificado, sino que los sistemas de valoración de los empleos se han hecho de espaldas a los saberes de las mujeres. El cuidado es visto socialmente como una aptitud inherente al hecho de ser mujeres, y por ello es algo que se aprehende sin pasar por el sistema educativo formal (Maruani, 1993). 
Tabla 3. Características del empleo en el sector de atención a la dependencia. España, 2009*

\begin{tabular}{|l|c|}
\hline \multicolumn{1}{|c|}{ Características del empleo } & $\%$ \\
\hline \multicolumn{2}{|c|}{ Incremento del empleo 2008-2009 } \\
\hline Todos los sectores de empleo & $-7,8 \%$ \\
\hline Asistencia en establecimientos residenciales & $+6,7 \%$ \\
\hline Actividades de servicios sociales sin alojamiento & $+10,8 \%$ \\
\hline \multicolumn{2}{|c|}{ Perión del sector 2009 } \\
\hline Asistencia en establecimientos residenciales \\
\hline Actividades de servicios sociales sin alojamiento \\
\hline \multicolumn{2}{|c|}{$82,8 \%$} \\
\hline Asistencia en establecimientos residenciales en el sector privado 2009 \\
\hline Actividades de servicios sociales sin alojamiento & $75,5 \%$ \\
\hline Actividades sanitarias & $76,5 \%$ \\
\hline Educación & $34,4 \%$ \\
\hline
\end{tabular}

(*) Los datos seleccionados corresponden al segundo trimestre del año.

Fuente: Elaboración propia a partir de datos EPA.

Todo ello va a tener su expresión específica en el sector del cuidado: expansión de un sector feminizado, precario y con un nivel alto de economía sumergida. En primer lugar parece claro que es un sector en clara expansión y que el sector público tiene un papel central en el fomento de este tipo de servicios, pero que éstos, al contrario que los otros grandes sistemas del Estado del Bienestar, están siendo gestionados por el sector privado (ver Tabla 3).

El sector que nos ocupa recibe influencias de todas las lógicas mercantiles y extramercantiles descritas. Es necesario hacer referencia al proceso histórico del sector para entender algunas de las lógicas actuales. En este sentido, el primer impulso del sector no proviene de la ampliación del Estado del Bienestar, sino que se promueve como una forma de fomentar el empleo femenino y luchar con las altas tasas de paro femenino de la primera década de los años noventa (Torns,1997). De hecho, se adecuaba a la política europea que tras el Libro Blanco de Delors había intensificado su impulso de los yacimientos de empleo, que intentaban fomentar el trabajo a nivel local para luchar contra las altas tasas de desempleo. Por tanto, la noción de bienestar estuvo lejos de la consideración de la expansión de este sector de empleo. En ese contexto, se buscaron soluciones para la contratación de mujeres, y se hallaron en un sector relativamente nuevo y que ante las previsiones demográficas de envejecimiento de la población se vieron como unas soluciones óptimas. No se pensó 
ni en la calidad del empleo ni en la calidad del servicio. En parte porque eran servicios-empleos que iban a dar respuesta a las demandas de una población excluida socialmente y silenciada, y en parte porque iban a emplear a mujeres que estaban en riesgo de exclusión social. El imaginario del servicio y la profesión se situó más en el discurso de la lucha por la cohesión social que en el de la calidad del empleo y del bienestar de las personas (Torns et al., 2009).

«(...) los servicios personales y los care services son un magnífico ejemplo de esta creciente feminización de los también llamados servicios pink collar. Unos empleos poco cualificados, con bajos salarios, y con una fuerte presencia de contratos temporales y a tiempo parcial. Lo que no hace sino mostrar la persistencia de la tradición de precariedad de la relación salarial en femenino». (Carrasquer y Torns, 2007)

Desde Francia, a mediados de los años noventa, surgen estudios sobre el significado y la naturaleza de los empleos de proximidad (Fraisse, 2000; Croff, 1996; Lallement, 2000), relacionando los distintos aspectos que intervienen en la configuración de este tipo de trabajo en empleo y representando una síntesis de todos los elementos que se han ido citando y que están implicados en la configuración del modelo de empleo del sector de servicios de proximidad, que estas autoras lo entienden desde la precariedad laboral (Fraisse, 1996 y 2000; Croff, 1996; Lallement 1996 y 2000). De ahí que consideren que el servicio de proximidad es la denominación dada al trabajo doméstico asalariado, y sitúan este proceso en un contexto de crisis de empleo (en el sentido de precariedad) y en relación con el trabajo invisible (Fraisse, 1996). Por otro lado, consideran también que las relaciones de dependencia, subordinación y dominación atribuidas al trabajo doméstico (que remiten a la idea de servidumbre) impiden que estas profesiones se conciban como profesiones cualificadas, conllevando esto a que las personas empleadoras se sitúen en una posición de superioridad respecto a las personas contratadas. El imaginario social sitúa a las personas encargadas de realizar estos trabajos más como siervas/criadas que como trabajadoras asalariadas, y el tipo de trabajos asignados a estos nuevos servicios de proximidad se asemejan al trabajo doméstico no asalariado y realizado bajo condiciones de subordinación.

Estas visiones nos permiten realizar alguna consideración en este campo, aunque los datos escasean podemos destacar que estos empleos, aun con el impulso de los nuevos marcos legales, cuando están en el mercado formal, no tienen buenas condiciones de trabajo (Aragón, Cruces y Rocha, 2008). No están bien remunerados, muchas veces son inestables, y no están reconocidos. Como expresa la cita de Fraisse que encabeza este artículo, se trata de empleos demasiado cercanos a los imaginarios serviles. Sin embargo, es cierto 
que el nuevo marco legal preveé un importante incremento del sector ${ }^{6}$. Lo que se desconoce es la vía de creación de empleo y en qué categorías se va a generar. Parece ser que el discurso político apuesta por la profesionalización, una demanda que también proclaman tanto los colegios profesionales, las organizaciones sindicales, e incluso las organizaciones patronales ${ }^{7}$ (Torns et al., 2009). Y, de hecho, el debate ahora está instalado en el cuerpo de competencias y el grado de formación que debe exigirse, puesto que aún persiste un sistema no estatal de formación profesional que no es equiparable a ningún grado medio ni superior de la Formación Profesional en España. Parece ser que la vía sería acercarse al sistema sanitario, un proceso inconcluso, pero que se plantea como la vía para la mejora del empleo.

\section{Conclusiones}

La comunicación recoge algunas reflexiones en torno a la temática del trabajo de cuidados y sobre todo propone una lectura desde el vínculo entre trabajo y bienestar. En concreto se ha plasmado una propuesta teórica basada en el estudio de los modelos nacionales de empleo. Se trata de un debate inconcluso, y lo que aquí se presenta son algunas pinceladas y planteamientos iniciales de esta perspectiva.

El cuidado toma relevancia, traspasando la academia para situarse ya en la arena política y pública. Este es el proceso que hemos querido demostrar en el primer apartado. Y recoger aquellos trabajos que desde el feminismo han servido para mostrar cuán importante es el cuidado para el bienestar de la población, denunciando que a veces esta dimensión continúa silenciada. Tras este breve recorrido por aquellas perspectivas que hoy en día permiten avanzar en el conocimiento del cuidado, se ha presentado una propuesta teórica para el estudio del empleo en el sector de atención a las personas. Este enfoque parte del contexto de los modelos nacionales de empleo, que explican las transformaciones en los modelos nacionales de empleo desde una perspectiva institucional. El empleo se inscribe así en un contexto social determinado, donde instituciones extramercantiles interactúan con el mercado, y es desde esta intersección desde donde se generan unas dinámicas nacionales u otras. Esto nos ha permitido dibujar un modelo nacional de empleo caracterizado por su precariedad e inestabilidad y fuertemente segmentado por razones de

6. Las previsiones sitúan en casi 300000 los nuevos empleos que se van a generar con el despliegue de la Ley de Dependencia.

7. Éstas conclusiones derivan de una investigación finalizada recientemente y realizada por el Centre de Recerca QUIT, y sobre la cual se está trabajando para elaborar publicaciones futuras. 
género. Un modelo que depende de la persistencia de una familia tradicional y un estado asistencialista. Es en este contexto donde hemos enmarcado el estudio del sector del cuidado. En este sentido los primeros indicios apuntan precisamente a que se trata de un sector de empleo incipiente que generará nuevos empleos incluso en un momento como el actual donde las tasas de desempleo son significativamente elevadas. Pero este empleo aún parece estar pensado desde la precariedad y desde el valor social obtenido por aquellos empleos próximos al trabajo de las mujeres. El tipo y la cobertura de los servicios nos dice que todavía estamos muy cerca de un planteamiento familista de la política social española y ello revierte claramente en el tipo de ocupación. La menor apuesta por servicios no ataca las necesidades reales de cuidado de las personas, tan sólo transmite los costes del cuidado a las familias, cubriendo éstas sus necesidades, ya sea vía la propia institución familiar o con soluciones privadas, unas decisiones que dependerán de la clase social y la capacidad económica mayor o menor a hacer frente al coste económico que suponen las soluciones privadas. De esta manera, parece que se pierde el tren de una política clara que apunte hacia la profesionalización del cuidado. En definitiva, nos encontramos ante un sector que genera ocupación precaria, altamente feminizado, desarrollado gracias al impulso de las políticas públicas y la expansión del sector privado en la gestión y prestación del servicio. Unas características que comparten algunos rasgos con los otros grandes sistemas de bienestar, pero que se alejan de ellos en tanto en cuanto las condiciones de empleo y el acceso a los servicios son precarias e insuficientes y que se asientan sobre un modelo persistente de male breadwinner.

A nuestro parecer, para revertir esta situación sería necesaria una apuesta por un modelo de organización social sensiblemente distinto al actual, implicando la exigencia de cambios en el ámbito de la división sexual del trabajo y en la orientación política respecto a la importancia de los servicios de atención a las personas. También exige cambios en el mercado, por ejemplo, en el campo de la revisión de las valoraciones de los puestos de trabajo o de las grandes escalas salariales. Sólo si se piensan cambios en este sentido, que alberguen a distintas esferas de la vida social de un país, se podrá pensar en un sector de atención a las personas como una apuesta definitiva hacia el bienestar, tanto de las personas que prestan los cuidados como de las que los reciben. Existen algunos países que parecen estar más cercanos a este modelo, de ahí que se argumente que estas reflexiones lejos de ser utópicas puedan ser realmente aplicadas. 


\section{Bibliografía}

Adelantado, J., Los cambios en el estado del Bienestar, Barcelona, Icaria, 2000.

Adelantado, J., J.A. Noguera y X. RAmbla, «El marco de análisis: las relaciones complejas entre la estructura social y políticas sociales», en Cambios en el Estado del Bienestar. Políticas sociales y desigualdades en España, J. Adelantado(coord..), Barcelona, Icaria-UAB, 2000, pp. 23-61.

Ahmed, A., «the Swedish elderly care», national Report Dynamics of National Employment Models (DyNAMO) project, Växjö: CAFO, Växjo University, 2006.

ANXO, D. y C. FAGAN, «The family, the state and now the market: the organisation of employment and Working time in home care services for the elderly», en Working in the Service Sector: a tale from different worlds, G. Bosch y S. Lehndorff (ed.), New York, Routledge, 2005, pp. 133-164.

ANXO, D. y H. NyMAN, «Home care in Sweden» en New forms of employment and working time in the Service Economy, E. Mermet y S. Lehndorff, Final Report, 2001.

- «Home care in Sweden» en Country report for the New Forms of Employment and Working Time in the Service Economy (NESY) Project, E. Mermet y S. Lehndorff, 2001.

AnXo, D., H. Nyman y C. FAGAN, «The elderly home care», New forms of Employment and Working Time in the Service Economy Conference, 26-27 de Abril de 2001, Bruselas.

ARAGON, J., J. CRUCES y F. ROCHA, Las condiciones laborales en el sector de atención a la dependencia: una aproximación a la calidad en el empleo, Madrid, Fundacion Primero de Mayo, 2008.

AstelarRa, J., «Marx y Engels y el movimiento de las mujeres. ¿Es posible una lectura feminista de Marx?, A priori, no 0, 1982.

BALBO L., «La doble presencia» en Las mujeres y el trabajo: rupturas conceptuales, C. Borderías, C. Carrasco y C. Alemany, Barcelona, Icaria-Fuhem, 1994, pp. 503-513.

BANYUlS, J. et al., «The Transformation of the Employment System in Spain: Towards a Mediterranean Neoliberalism?» en European Employment Models in Flux. A comparison of Institutional Change in Nine European Countries, G. Bosch, S. Lehndorff y S. Rubery, Basingstoke, Palgrave Macmillan, 2009, pp. 247-270.

BENERIA, L., «Reproducción, producción y división sexual del trabajo», en Mientras Tanto, 6, 1981.

Bettio, F. y J. Plantenga, «Comparing care regimes in Europe», en Feminist Economics, n. ${ }^{\circ} 10$ (1), 2004, pp. 85-113. 
BetTiO, F; A. SimONAZzi y P. Villa, «Change in care regimes and female migration: the "care drain" in the Mediterranean», en Journal of European Social Policy, n. ${ }^{\circ} 16$ (3), 2006, pp. 271-285.

Borderías, C., C. CARrasco y C. Alemany, Las mujeres y el trabajo: rupturas conceptuales, Barcelona, Icaria-Fuhem, 1994.

BORRÁs, V., S. MORENO y C. RECIO, «La incorporación de los hombres en la esfera doméstica», en Sociología del Trabajo, n. ${ }^{\circ}$ 67, 2009, pp. 97-116.

Bosch, G., S. LeHndorfF y J. Rubery, (ed.), European Employment Models in Flux. A comparison of Institutional Change in Nine European Countries, Basingstoke, Palgrave Macmillan, 2009.

- «European Models in Flux: Pressures for change and Prospects for survival and Revitalisation» en European Employment Models in Flux. A comparison of Institutional Change in Nine European Countries, en G. Bosch, S. Lehndorff y S. Rubery (eds.), Basingstoke, Palgrave Macmillan, 2009, pp. 1-57.

Carrasco, C., A. Alabart, y M. Mayordomo, Mujeres, trabajos y políticas sociales: una aproximación al caso español, Madrid, Instituto de la Mujer, 1997.

CARRASQUer, P. y T. TORNS, «Cultura de la precariedad: Conceptualización, pautas y dimensiones. Una aproximación desde la perspectiva de género», en Sociedad y Utopía, n. ${ }^{\circ}$ 29, 2007, pp. 139-156.

Carrasquer, P., T. TORns, E. Tejero y A. Romero, «El trabajo reproductivo», en Papers: Revista de Sociologia, n. ${ }^{\circ}$ 55, 1998, pp. 95-144.

COATES, D. (ed.), Varieties of Capitalism, Varieties of Approaches, Basingstoke, Palgrave-Macmillan, 2005.

CROFF, B. , « Les emplois familiaux:le travail domestique réhabilité? », en Cahiers du Mage, n. ${ }^{\circ} 4,1996$, pp. 77-82.

DALY, M. y J. LEWIS, «The concept of social care and the analysis of contemporary welfare states», British Journal of Sociology, n 51, (2), 2000, pp. 281-298.

EsPing-Andersen, G., The three worlds of welfare capitalism, Cambridge, Polity Press, 1990.

EVERTSSON, L, «The Swedisch Welfare State and the emergente of Female State Occupations», en Gender, Work and Organisations, 7 (4), 2000, pp.230-241.

FAGAN, C. y D. NiXON, «Home care in the United Kingdom», Country report for the New Forms of Employment and Working Time in the Service Economy (NESY) Project, Manchester: Department o Sociology, University of Manchester, 2000.

FINCH, J., «The politics of comunity care in Britain» en Gender and caring, C. Ungerson, Hertfordshire, Harvester Wheatsheaf, 1990, pp. 34-58.

FinCH, J. y D. GROVES, A labour of love: Women, work and caring, Londres, Routdlege and Kegan Paul, 1983. 
FRAISSE, G., «Servidumbre, servicios de proximidad y democracia» en Las nuevas fronteras de la desigualdad, M. Maruani, Ch. Rogerat y T. Torns, Barcelona, Icaria, 2000, pp. 227-232.

GARDINER, J., «Rethinking self-sufficiency: employment, families and welfare», en Cambridge Journal of Economics, n. ${ }^{\circ}$ 24, 2000, pp. 671-689.

GRAHAM, H., «Caring: a labour of love» enA labour of love: women, work and caring, J. Finch y D. Groves, London, Routledge and Kegan Paul, 1983, pp.13-30.

Hochschild, A. R., La mercantilización de la vida intima. Apuntes de la casa y el trabajo, Buenos Aires, Katz Editores, 2008.

IMSERSO, Cuidados a las personas en los hogares españoles. El entorno familiar, [en línea], MTAS-IMSERSO Madrid, 2005, <http://www.imserso.es/Presentacion/ groups/imserso/documents/binario/cuidadosppmmhogares.pdf>.

LALLEMENT, M., «Familia y empleos de servicio» en Las nuevas fronteras de la desigualdad, M. Maruani, Ch. Rogerat y T. Torns, Barcelona, Icaria, 2000, pp. 249-268.

LETABLIER, M.-TH., «El trabajo de "cuidados" y su conceptualización en Europa», en Trabajo, género y tiempo social, C. Prieto, Madrid, Hacer, 2007, pp. 64-84.

LEWIS, J.(ed.), Gender, Social Care and Welfare State Restructuring in Europe, Aldershot, Ashgate, 1998.

LOPE, A., A. RECIO y F. GIBERT, La qualitat de l'ocupació i dels serveis d'assistència domiciliaria a Manlleu, Barcelona, QUIT, 2004.

LOPE, A., F. GIBERT y A. RECIO, Propostes per l'establiment d'un protocol sobre ocupació en la prestació dels serveis d'Atenció Domiciliaria de Manlleu, Barcelona, QUIT, 2005.

MARUANI, M., «La cualificación, una construcción social sexuada», en Revista de economía y sociología del trabajo, n. ${ }^{\circ} 21-22,1993$, pp. 41-49.

MiguÉlez, F., «La flexibilidad laboral», en Trabajo, n. ${ }^{\circ}$ 13, 2004, pp. 17-36.

Miguélez, F. y C. PRIETo, Las relaciones de empleo en España, Madrid, Siglo XXI, 1999.

Miguélez, F. A., Lope y I. OlivareS, «Eldery care sector in Spain», en Proyecto Dynamo, (impreso), Barcelona, QUIT, 2006.

Muñoz DE Bustillo, R., «Retos y restricciones del Estado del Bienestar en el cambio de siglo«, en El Estado del Bienestar en el cambio de siglo, R. Muñoz de Bustillo (ed.), Madrid, Alianza, pp. 50-108.

PASTOR, I., « ¿Treball públic amb lògica privada?» en Revista de l'Associació Catalana de Sociología, n. ${ }^{\circ}$ 15, 2001, pp. 154-171.

- ¿La gestión privada en la Administración Pública? El caso de los servicios hospitalarios de Cataluña, Madrid, Consejo Económico y Social, 2006.

PICCHIO, A., «El trabajo de reproducción, tema central en el análisis del mercado laboral», en Las mujeres y el trabajo. Rupturas conceptuales, C. Borderías, C. Carrasco y C. Alemany, Barcelona, Icaria-Fuhem, 1994, pp. 450-490. 
RECIO, A., «Low pay in Spain», en Transfer, 7(2), 1999, pp. 321-337.

— «Low pay in Spain», en Transfer, vol. 7, 2, 2001, pp. 321-337.

— «El negoci de la dependència» en Carrer, 111, 2009, p. 16.

Rodríguez CABrero, G. (comp.), Estado, privatización y bienestar. Un debate de la Europa Actual, Barcelona, Icaria-Fuhem, 1991.

- «Los restos de la política social en la era de la globalización» en La sociedad: Teoría e investigación empírica, AA.VV., Madrid, CIS, 2002, pp. 507-536.

RUBERY, J., «What do women want from full employment?», en Working for Full Employment, J. Philpott, Londres, Routdlege, 1997, pp. 63-80.

Rubery, J., M. SMITH y C. FAGAN, Women and European Employment. Trends and prospects, Londres, Routledge, 1999.

RUBERY, J., G. BOSCH y S. LEHNDORFF, The influence of the EU on the evolution of National Employment Models, International Labor Organization-International Institute for Labour Studies, Ginebra, 2008.

SAInSBURY, D., Gendering welfare states, Sage Londres, Publications, 1996.

SARACENO, CH., «Familismo ambivalente y clientelismo categórico en el Estado del Bienestar italiano», en El estado del Bienestar en la Europa del Sur, L. Moreno y S. Sarasa (coord.), Madrid, CSIC, 1995, pp. 261-288.

Simonazzi, A. M., "Care regimes and nacional employment models», Cambridge Journal of Economics, n. ${ }^{\circ} 33,2009$, pp. 211-232.

Thomas, C., «De-constructing concepts of care», en Sociology, n. 27 (4), 1993, pp. 649-669.

TORNS, T., «Los servicios de proximidad ¿un yacimiento de empleo?», en Revista de Treball Social, 1997, pp. 40-53.

- «Las asalariadas: un mercado con género», en Las relaciones de empleo en España, F. Miguélez y C. Prieto, Madrid, Siglo XXI, 1999, pp. 150-161.

- «El trabajo y el cuidado: cuestiones teórico-metodológicas desde la perspectiva de género», en Empiria, n. ${ }^{\circ}$ 15, 2008, pp. 53-73.

— «El futuro del empleo femenino y los yacimientos de empleo», en La mujer en el mercado de trabajo, P. Rodríguez y otros, Instituo de Desarrollo Regional, Málaga, 2000, pp. 223-243.

TORNS, T. et al., Local development and care services in Catalonia, comunicación, 27th International Labour Process Conference «Work Matters», Edimburgo 6,7 y 8 de abril de 2009.

UnGERSON, C., "The language of care. Crossing the boundaries», en Gender and caring. Work and Welfare in Britain and Scandinavia, C. Ungerson, Hertfordshire, Harever Wheatsheat, 1990, pp. 9-33. 